\title{
Bibliotheksentwicklung an der Universität Zürich als Standortoptimierung: Gebietsplanung und Bibliotheksarrondierung als Chance
}

\begin{abstract}
Für die Universität Zürich als grosse Stadtuniversität ist das Gebietsmanagement im Hochschulgebiet Zürich Zentrum und die Entwicklungsplanung von Bibliotheksstandorten eine vielschichtige Aufgabe. Der Beitrag beleuchtet die aktuelle Situation historisch und systematisch. Das geplante Lehr- und Lernzentrum im Neubauvorhaben FORUM UZH auf dem zentral gelegenen Areal Wässerwies ist ein aktueller Referenzpunkt, an dem die Gebietsplanung allgemein wie auch die Planung eines modernen Lehr- und Lernzentrums mit ihren vielschichtigen strukturellen Anforderungen und die aktuellen Herausforderungen der Bibliotheksentwicklung an der UZH zusammentreffen. Das Bauvorhaben FORUM UZH bietet mit seiner Realisierung voraussichtlich im Jahr 2026 eine zentrale Chance für die Weiterentwicklung der Bibliothekslandschaft der UZH.
\end{abstract}

\section{Einleitung}

Die Entwicklung von Lernzentren und Bibliotheken steht auch an der Universität Zürich (UZH) komplexen Herausforderungen gegenüber: umfassende Digitalisierung, veränderte Lehr- und Lernformen, steigende Anzahl an Studierenden und Forschenden, Konzentration von Standorten mit Aufgabe von kleineren Liegenschaften und Planung von Neubauprojekten im Rahmen von übergeordneten Gebietsplanungen. Der vorliegende Beitrag skizziert die Entwicklung der Gebäudeinfrastruktur und die Bibliotheksentwicklung der UZH. Das historisch gewachsene mehrschichtige Bibliothekssystem mit einer Vielzahl an Standorten trifft auf grosse Bauvorhaben und Arrondierungsprozesse, die in jüngster Zeit deutlich an Dynamik gewonnen haben. Die aufwendige Schaffung von hoheitlichem Planungsrecht und verhältnismässig langen Planungs- und Bewilligungszeiten grosser Bauprojekte im öffentlichen Sektor stehen meist in Spannung mit den eher kurz- und mittelfristigen Anforderungen im Wissenschaftsbetrieb mit einer sehr dynamischen Forschung und Lehre. Das Bauvorhaben Bildungs- und Forschungszentrum FORUM UZH, das auf dem zentralen Areal Wässerwies im Hochschulgebiet Zürich Zentrum bis 2026 entstehen soll, ist ein aktuelles Bei- 
spiel für die Herausforderungen der baulich-akademischen Hochschulentwicklung. So sind raum- und bauplanerische Erfordernisse mit der bibliothekarischen Strategie als einem Teilbereich der Gesamtplanung zukunftsorientiert in Beziehung zu setzen.

\section{Historischer Abriss: zentrale Schritte der baulichen Entwicklung und der Bibliotheksentwicklung}

Die Universität Zürich ${ }^{1}$ wurde 1833 gegründet und erhielt, nach einem Provisorium im Gebäude des Polytechnikums (heute ETH Zürich), 1914 ihr von den Architekten Karl Moser und Robert Curjel geplantes Kollegiengebäude in prominenter Stadtlage in direkter Nachbarschaft zum Hauptgebäude der ETH Zürich - bis heute das zentrale Gebäude der UZH im Zentrum. Durch das Wachstum der UZH in den folgenden Jahrzehnten entwickelte die UZH im Zentrum eine „quartierübergreifende Grossstruktur“2 mit einer zunehmenden Zahl von grösseren Gebäuden und Immobilien in Streulagen. Die begrenzten Entwicklungsmöglichkeiten im Zentrum und die steigenden technischen Anforderungen der naturwissenschaftlichen Fächer führten ab 1962 zu Planungen für einen Campus auf dem Strickhofareal in Zürich Irchel, welcher in drei grossen Etappen 1979, 1983 und 1994 erstellt wurde. ${ }^{3}$ Da auf dem Campus Irchel mit einer 4. Etappe nicht ausreichend experimentelle Flächen zur Verfügung gestellt werden konnten, erweiterte die UZH 2013 am Ausweichstandort Schlieren bei Zürich ihre Laborkapazität mit Mietflächen. Derzeit wird am Standort Irchel in einer 5. Etappe ein grosses Laborgebäude mit dem Schwerpunkt Chemie erstellt. Raumnot aufgrund zurückhaltender kantonaler Investitionen in Grossprojekte herrscht auch am

1 Vgl. den Überblick bei Sebastian Brändli: Universität Zürich. In: Historisches Lexikon der Schweiz. 12 Bde. Basel 2002ff. Bd. 12, S. 626-627.

2 Caspar Schärer: Bauten. Moser, Ziegler, Calatrava. In: Universitätsleitung der Universität Zürich (Hrsg.): Rückblenden, Einsichten, Ausblicke. Wissen teilen. 175 Jahre Universität Zürich. Zürich 2008, S. 119-124, hier S. 120.

3 Die veterinärmedizinische Fakultät (gegründet 1902, heute Vetsuisse Fakultät UZH) mit Tierspital war schon seit 1963 auf dem Areal der kantonalen Landwirtschaftlichen Schule Strickhof in der Nähe des Irchel untergebracht (vgl. Konrad Zerobin: Die Veterinärmedizinische Fakultät seit ihrer Gründung im Jahr 1902. In: Rektorat der Universität Zürich (Hrsg.): Die Universität Zürich 1933-1983. FS zur 150-Jahr-Feier der Universität Zürich (Gesamtredaktion Peter Stadler). Zürich 1983, S. 445-470, hier S. 450. 
Standort im Zentrum. Daher mussten 2006 von der UZH in Zürich Oerlikon als Ausweichstandort vor allem für sozialwissenschaftliche Fachbereiche Büro-, Bibliotheks- und Lehrflächen angemietet werden. ${ }^{4}$

Die historische Bibliotheksentwicklung der Universität folgt in grossen Linien der Standortentwicklung. Bereits Vorläufer der UZH, wie das Collegium Carolinum als theologische Lehranstalt und verschiedene wissenschaftliche Gesellschaften, verfügten über eigene Bibliotheken. Mit der Universitätsgründung begann der Aufbau von wissenschaftlich orientierten Beständen an verschiedenen Standorten und im neuen Kollegiengebäude waren und sind mehrere Institutsbibliotheken eingebunden. Eine für die Bibliotheksentwicklung der Universität wichtige Entwicklung begann mit der Eröffnung der Zentralbibliothek Zürich (ZB) 1917 als Zusammenschluss von Kantonsbibliothek und Stadtbibliothek. Die 1835 aus verschiedenen Vorläuferbibliotheken (insbesondere des Collegium Carolinum) gegründete Kantonsbibliothek umfasste auch Bestände der Universität. Mit ihrem 1917 in günstiger Lage zur Universität im Zentrum am Zähringerplatz eröffneten Neubau konnte sich die Zentralbibliothek als „Hauptbibliothek des universitären Bibliothekssystems für die Geisteswissenschaften“5 positionieren. Aus der Stadtbibliothek übernahm sie zentrale Elemente der historischen Sammlungen, die bis heute einen zentralen Stellenwert an der ZB einnehmen. Diese Fusion unterschiedlicher Trägerschaften ist auch der Hintergrund für die bis heute gültige Rechtsform der ZB als einer von Stadt und Kanton getragenen Stiftung, auf deren Gebäudeentwicklung die UZH allerdings keinen direkten Einfluss hat. Die ZB entwickelte sich dynamisch zu einer der grössten Bibliotheken der Schweiz mit geistes- und sozialwissenschaftlichem Schwerpunkt weiter und konnte 1995 mit einem grossen Erweiterungsbau im Magazin- und im Publikumsbereich am bisherigen Standort ${ }^{6}$ und neuerdings mehreren Aussenlagern ${ }^{7}$ erweitert werden. Mit heute rund 800 Selbststudiums-

4 Vgl. Schärer, Bauten (wie Anm. 2), S. 120 ff. Hans Peter Stucki, Michael Ganz: Meilensteine der Universitätsgeschichte. In: Universitätsleitung der Universität Zürich (Hrsg.), Rückblenden (wie Anm. 2), S. 127-134.

5 Roland Mathys: 1629 Stadtbibliothek - Zentralbibliothek 1979. Zürich 1979, S.12. Vgl. Rea Brändle: Sich Treffen, Lernen, Verweilen. Die Zentralbibliothek als öffentlicher Raum. In: Rea Brändle, Markus Brühlmeier u.a.: Wissen im Zentrum. 100 Jahre Zentralbibliothek Zürich. Zürich 2017, S. 19-72, hier S. 20-32.

6 Der Erweiterungsbau erfolgte nach jahrzehntelanger Diskussion und Planung. Vgl. Zentralbibliothek Zürich (Hrsg.): Die Zentralbibliothek baut. Texte und Bilder. Schriftleitung Roland Mathys. Zürich 1996. Vgl. ferner Brändle, Sich Treffen (wie Anm. 5), S. 54-64.

7 Die ZB ist Partnerin der Kooperativen Speicherbibliothek (s.u.) und betreibt in Oetwil am See (Kanton Zürich) seit 2016 ein weiteres grosses Aussenlager. 
plätzen ${ }^{8}$ und zahlreichen Ausleihen stellt sie eine zentrale Einheit der Literaturversorgung für die Universität Zürich dar.

Mit der Entwicklung der Universität, dem fortschreitenden Wachstum der Studierendenzahlen, das sich insbesondere seit den 60er Jahren des 20. Jahrhunderts deutlich verstärkt hat, und den zahlreichen zusätzlichen Standorten besonders im Zentrum begann, wie an anderen Stadtuniversitäten, der Aufbau zahlreicher Instituts- und Seminarbibliotheken.

1980 wurde die heutige Hauptbibliothek der Universität Zürich zunächst als Koordinationsstelle auf dem neuen Campus Irchel errichtet und im Gebäude der ehemaligen Landwirtschaftsschule Strickhof untergebracht. ${ }^{9}$ Sie erhielt 1995 ein zusätzliches Gebäude ebenfalls auf dem Campus Irchel, die heutige Hauptbibliothek - Naturwissenschaften. 2004 wurde ihr zudem die heutige Hauptbibliothek - Medizin Careum ${ }^{10}$ im Zentrum organisatorisch angegliedert. Sie führt heute drei grosse Standorte mit rund 1'000 Selbststudiumsplätzen. Mit dem Neubau der Bibliothek des Rechtswissenschaftlichen Seminars (2004), mit 560 Selbststudiumsplätzen die grösste Einzelbibliothek der UZH, mit der Einrichtung der Bibliothek des Asien-Orient-Instituts (2013) oder neuerdings mit dem Erweiterungsbau Betriebswirtschaft (Plattenstrasse, Eröffnung 2018) setzt die UZH weitere bibliotheksbezogene Akzente im Baubereich.

Zudem führt die UZH weitere bedeutende Institutsbibliotheken, z.B. das Romanische Seminar, das Deutsche Seminar oder das Historische Seminar. Sie verfügen neben Selbststudiumsplätzen auch über beachtliche Bestände vor Ort. Angegliedert sind häufig kleinere Bibliotheken an Aussenstandorten. ${ }^{11}$ Daneben

8 Der Begriff Selbststudiumsplätze umfasst als Überbegriff Einzel- wie auch Gruppenarbeitsplätze in Ruhe- und Gesprächszonen. Er wird als Gegenbegriff zu Studierendenplätzen im Bereich Kontaktstudium, wie Hörsäle und Seminarräume, verwendet.

9 Vgl. Wilfried Lochbühler: Dreischichtigkeit zwischen Diversifikation und Kooperation: Das Bibliothekssystem der Universität Zürich. In: Konstanze Söllner, Wilfried Sühl-Strohmenger (Hrsg.): Handbuch Hochschulbibliothekssysteme. Leistungsfähige Informationsinfrastrukturen für Wissenschaft und Studium. Berlin/Boston 2014, S. 112-120, hier S. 115f.; Markus Brühlmeier: Verzetteln und Vernetzen. Vom analogen zum digitalen Katalog. In: Brändle/Brühlmeier u.a.: Wissen (wie Anm. 5), S. 73-121, hier S. 100-105.

10 Vgl. Wilfried Lochbühler: Hauptbibliothek - Medizin Careum in Zürich. Integrale Literaturversorgung für Medizin und Gesundheitsberufe. In: Rafael Ball, Stefan Wiederkehr (Hrsg.): Vernetztes Wissen. Online. Die Bibliothek als Managementaufgabe. Festschrift für Wolfram Neubauer zum 65. Geburtstag. Berlin/Boston 2016, S. 313-326.

11 So führt das Rechtswissenschaftliche Institut drei, das Historische und das Theologische Seminar jeweils zwei Bibliotheken an Nebenstandorten (vgl. http://www.uzh.ch, die Seiten der jeweiligen Institute und Seminare). Einen Überblick zum Bibliotheksnetz der UZH bieten auch die detaillierten Zahlen der Schweizerischen Bibliotheksstatistik: http://www.bfs.admin.ch (Rubrik: 
gibt es zahlreiche kleinere Institutsbibliotheken mit Schwerpunkt in der Philosophischen Fakultät (Zentrum, Oerlikon), bedingt durch Streulagen aber auch an anderen Fakultäten. Insgesamt verfügt die UZH über 99 bibliothekarische Standorte mit Buchbeständen an 67 Gebäudestandorten, die von rund 45 verschiedenen Einrichtungen (Institute, Seminare, Fakultäten, zentrale Einheiten) geführt werden. $^{12}$

Die Hauptbibliothek der UZH nimmt zunehmend gesamtuniversitäre Aufgaben wahr, z.B. im Bereich Bibliotheksverwaltungssystem (in Zusammenarbeit mit dem NEBIS-Verbund), E-Medien-Verwaltung, Open Access/Publikationsunterstützung oder Kooperative Speicherbibliothek. Sie ist zentral dem Prorektorat Rechts- und Wirtschaftswissenschaften unterstellt und vertritt die UZH auch nach aussen in Kooperationen und Gremien. Die Instituts-, Seminar- und Fakultätsbibliotheken der Universität fungieren als Literaturversorgungs- und Lernzentren vor Ort und sind im Blick auf Literaturverwaltung und Benutzungsmanagement organisatorisch selbständig. Sie unterstehen den jeweiligen Instituts- oder Fakultätsleitungen und bilden mit ihrer besonderen Nähe zu Dozierenden und Studierenden insbesondere in der Philosophischen Fakultät ein bedeutendes Element ihrer Identität. ${ }^{13}$ Das (einschliesslich ZB) faktisch dreischichtige Bibliothekssystem der $\mathrm{UZH}^{14}$ ist für die Gesamtsteuerung wie auch im Hinblick auf Arrondierungen, Gebäudeplanungen und Standortentwicklungen der UZH insgesamt von zentraler Bedeutung und steigert die Komplexität dieser Prozesse erheblich.

\section{Arrondierungen und Optimierungen von Bibliotheksstandorten: aktuelle Entwicklungen}

In den zurückliegenden Jahren zeichnete sich zunehmend ein Trend zur Optimierung, Zusammenlegung und Neuplanung von Bibliotheksstandorten an der UZH

Kultur, Medien, Informationsgesellschaft, Sport). Alle Links in diesem Beitrag wurden am 26.03.2018 überprüft.

12 Quelle: Standortverzeichnis der UZH der Abteilung Immobilienentwicklung (internes Dokument).

13 Neben Medizin/Naturwissenschaften, die zentral durch die Hauptbibliothek versorgt werden, haben die Theologische Fakultät, die Rechts- und die Wirtschaftswissenschaftliche Fakultät sowie die Vetsuisse Fakultät die Bibliotheken bereits vollständig (oder weitgehend) organisatorisch der Fakultätsebene zugeordnet.

14 Vgl. Lochbühler, Dreischichtigkeit (wie Anm. 9), S. 116-120. 
ab. Einerseits ist der Wandel hin zur vermehrt digitalen Bibliothek besonders im Bereich der Naturwissenschaften und der Medizin weit fortgeschritten. Andererseits nimmt durch die Entwicklung der UZH der Raumbedarf ständig zu.

So läuft schon seit Jahren ein Prozess der Konsolidierung bei den zahlreichen Klinikbibliotheken innerhalb des Universitätsspitals Zürich, die fortschreitend aufgelöst und mit ihrem dedoublierten Bestand in die Hauptbibliothek - Medizin Careum integriert werden. Entsprechend dem Gesamttrend zur digitalen Medienversorgung hat bereits in den 90er Jahren auf dem naturwissenschaftlich geprägten Campus Irchel die Integration und Dedoublierung der gedruckten Bestände der ehemals zahlreichen Institutsbibliotheken begonnen, der inzwischen weit fortgeschritten ist. ${ }^{15}$ Ab 2012 wurden zudem Bestände der früheren Studienbibliothek Irchel deutlich reduziert und in die Hauptbibliothek - Naturwissenschaften verlagert. Damit konnten dringend benötigte zusätzliche Leseplätze geschaffen und 2014 ein modernes Lernzentrum auf dem Campus Irchel eingerichtet werden. Das Institut für Informatik hat 2015 seine Institutsbibliothek physisch weitgehend aufgelöst und die Bestände der Hauptbibliothek - Naturwissenschaften übergeben.

Aber auch in stärker an gedruckten Beständen orientierten Fachdisziplinen nehmen Arrondierungen zu, insbesondere vor dem Hintergrund der angespannten Raumsituation der UZH. So wurde 2014 die Bibliothek des Instituts für Mathematik in die Hauptbibliothek - Naturwissenschaften integriert, der Bestand optimiert und damit gewonnene Flächen im Institutsgebäude auf dem Campus Irchel für anderweitige Nutzung zur Verfügung gestellt.

Ein aktuelles Beispiel ist ferner die im Jahr 2017 abgeschlossene Integration der Bibliothek für Medizingeschichte (bisher Hirschengraben im Zentrum) in die Hauptbibliothek - Medizin Careum (Gloriastrasse im Zentrum). Ihre Bestände wurden reduziert, dedoubliert, verlegt und die Versorgung erfolgt nun zentral. Entscheidende Voraussetzung dafür war 2014 der Beitritt der UZH zum Verein Kooperative Speicherbibliothek Schweiz ${ }^{16}$, an dem verschiedene Universitätsund Kantonsbibliotheken beteiligt sind. ${ }^{17}$ Er betreibt in Büron (Kanton Luzern)

15 Die Einrichtung eines zusätzlichen zentralen Bibliotheksgebäudes 1995 auf dem Campus Irchel (die heutige Hauptbibliothek - Naturwissenschaften) erfolgte auch vor diesem Hintergrund. Aktuell verfügen dort noch das Geographische Institut und das Anthropologische Institut über eigene Fachbibliotheken.

16 Mitglieder im Verein Speicherbibliothek sind derzeit, neben der Universität Zürich und der Zentralbibliothek Zürich, die Zentral- und Hochschulbibliothek Luzern, die Universitätsbibliothek Basel, die Zentralbibliothek Solothurn und die Bibliothek der Universität St. Gallen (HSG).

17 Vgl. hierzu den Beitrag „Der weite Blick“ von Ulrich Niederer in diesem Band; ferner: Ulrich Niederer: Aufbewahren in Bibliotheken - konzentrieren oder verteilen? Beides! Ein Bericht aus der Schweiz. In: 027.7. Zeitschrift für Bibliothekskultur 3/1 (2015), S. 4-11. 
ein 2016 eröffnetes, teilautomatisiertes Lager für Bücher (Kapazität derzeit rund 3 Mio. Bände) inklusive elektronischem Dokumentenlieferdienst und Logistikstrukturen zum Buchversand. Erst durch Dedoublierung und Auslagerung insbesondere von medizinischen Zeitschriftenbeständen aus der Hauptbibliothek Medizin Careum in die Speicherbibliothek konnte Raum zur Integration der Bestände der Bibliothek Medizingeschichte geschaffen werden. Auch verschiedene Institutsbibliotheken der UZH haben bereits die Möglichkeiten der Speicherbibliothek für Auslagerungen und Flächenoptimierung genutzt. Derzeit laufen an der HBZ Planungen zur Integration der Bibliothek des Geographischen Instituts (mit umfangreichen Beständen) in die Hauptbibliothek - Naturwissenschaften, deren naturwissenschaftliche Zeitschriftenbände zuvor in die Kooperative Speicherbibliothek ausgelagert werden müssen, um die nötigen Flächen für die Übernahme der geographischen Bestände zu schaffen.



Abb. 1: Hauptbibliothek - Medizin Careum Zürich (@ Frank Brüderli)

Die bisherigen Erfahrungen mit Arrondierungen und organisatorischen Zusammenlegungen von Bibliotheken zeigen summarisch folgende Vorteile auf:

1. Mit Arrondierungen von Bibliotheken können knappe Flächen in Stadtlagen freigespielt und optimiert genutzt werden. Da Arrondierungen meist mit einer Überprüfung des gedruckten Bestandes und der Möglichkeit zur Dedoublie- 
rung einhergehen, reduziert sich insgesamt der Bedarf an Flächen für Bibliotheksbestände.

2. Insbesondere die Auslagerung von Beständen in die Kooperative Speicherbibliothek verstärkt diese Möglichkeiten. In der Speicherbibliothek werden Zeitschriftenbestände in einem kollektiven Bestand gesammelt und auf ein gemeinsames Exemplar dedoubliert, sodass das Gesamtvolumen deutlich sinkt. Zusätzlich betragen die Archivierungskosten pro Band in der Speicherbibliothek nur einen Bruchteil der Kosten einer Archivierung in hochpreisigen Stadtlagen wie in der Stadt Zürich. ${ }^{18}$

3. Arrondierungen bieten betriebliche Vorteile, da die Einbindung von kleineren Fachbibliotheken in grössere Einheiten attraktivere Serviceleistungen ermöglicht (z.B. erweiterte Öffnungszeiten, optimierte Beratungsdienste, Ausleihe durch Selbstverbuchung).

4. Die Integration in grössere Einheiten fördert die Entstehung moderner Lernzentren und die Schaffung zusätzlicher Selbststudiumsplätze. Bei gleichzeitiger Aufhebung von Bibliotheksstandorten sollte die Gesamtzahl der verfügbaren Selbststudiumsplätze nicht reduziert und wo möglich erhöht werden.

5. Personelle Ressourcen können in grösseren Betriebseinheiten optimiert eingesetzt werden. Insbesondere in der Benutzung können bisher redundante Theken- und Beratungsdienste zusammengeführt werden. Aber auch in der Katalogisierung oder im Bereich Informationskompetenz entstehen Synergien. Bei neuen Dienstleistungen (wie Publikationsunterstützung, Forschungsdatenmanagement, Informationskompetenz) bilden erst grössere Einheiten die Voraussetzung für eine ausreichende Professionalisierung.

6. Damit unterstützen Arrondierungen mittelbar die notwendige personelle Entwicklung bibliothekarischer Dienstleistungen hin zu zukunftsgerichteten Aufgabenfeldern, die angesichts beschränkter Budgets nicht allein durch zusätzliche Anstellungen abgedeckt werden können. Diese Entwicklung muss, über die Standortoptimierung hinaus, in eine Gesamtstrategie und gesamtuniversitäre Personalentwicklungsplanung für Bibliotheken eingebunden sein.

Insbesondere die grössere räumliche Distanz zwischen dem Arbeitsort der Forschenden und den Bibliotheksbeständen wird allerdings häufig als gravierender Nachteil von Arrondierungen gesehen. Am Standort Irchel zeigt die Erfahrung,

18 Erfahrungen in amerikanischen Speicherbibliotheken sind eindeutig. Die Archivierungskosten pro Band und Jahr liegen Onsite bei 4,26 USD, Offsite in einer Speicherbibliothek bei 0,86 USD (vgl. University Leadership Council: Redefining the Academic Library. Managing the Migration to Digital Information Services. Washington 2011, S. 51). Für Liegenschaften in der Stadt Zürich bzw. die Speicherbibliothek bewegen sich die Kosten in einer ähnlichen Grössenordnung. 
dass durch die räumliche Nähe der Hauptbibliothek - Naturwissenschaften zu den Institutsgebäuden diese Distanz nicht als gravierender Nachteil betrachtet wird. Bei grösseren Distanzen zwischen Bibliothek und Institut, z. B. im Fall der Medizingeschichte, wurden ein täglicher Kurierdienst für gedruckte Bestände, ein elektronischer Dokumentenlieferdienst und ein zusätzlicher Handapparat im Institutsgebäude eingerichtet. Aus der Speicherbibliothek können Monographien per Kurier und Zeitschriftenartikel via elektronischen Dokumentenlieferdienst innert 24 Stunden geliefert werden. Dass nicht mehr alle gedruckten Bestände direkt vor Ort verfügbar sind, wird besonders im geisteswissenschaftlichen Bereich gleichwohl auch als Verlust angesehen. Wenn keine elektronische Ausgabe vorhanden ist, entfällt z.B. das Browsen am Regal oder das Durchblättern von Zeitschriftenbänden, da die Literatur jeweils erst via Bibliothekssystem bestellt und geliefert werden muss. ${ }^{19}$

Arrondierungen und Zusammenführungen von Bibliotheken sind anspruchsvolle Projekte, die in der Regel eine gesonderte Finanzierung und damit eine Investition erfordern. Dedoublierungs- und Katalogisierungsarbeiten, Transport, Infrastrukturanpassungen in der aufnehmenden Bibliothek wie zur Nachnutzung frei werdender Flächen und die Einrichtung zusätzlicher Dienstleistungen (z.B. Logistik, Dokumentenlieferdienst) bedürfen projektorientierter Steuerung und Finanzierung. Nicht unterschätzt werden sollten Personalentwicklungsmassnahmen bei der Zusammenführung von Teams und die entsprechende Herausforderung für die Führung.

19 Vgl. auch Andreas Ledl, David Tréfas: Speicherbibliotheken: eine Nachlese. In: 027.7. Zeitschrift für Bibliothekskultur 3/1 (2015), S. 20-29, hier S. 24. 


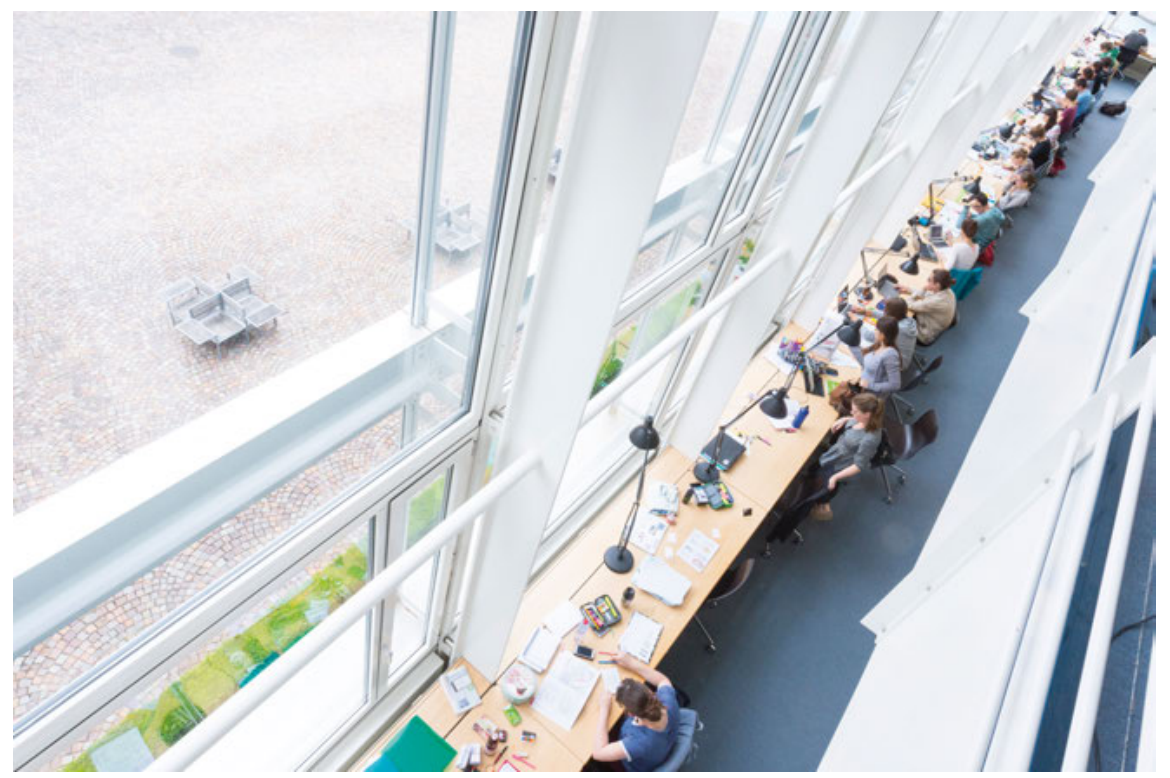

Abb. 2: Hauptbibliothek - Naturwissenschaften Campus Irchel Zürich (৫ Frank Brüderli)

\section{Theorie: Organisations- und Planungstheorie in der baulichen Hochschulentwicklung (Situation des öffentlichen Bauens)}

Bauliche Hochschulentwicklung und somit auch der Neubau von Lernzentren und Bibliotheken arbeitet im Differenzbereich von mindestens zwei Verständnissen von Organisation. Auf der einen Seite das auf Freiheit angelegte Wissenschaftssystem mit Forschung und Lehre und auf der anderen Seite das auf Regulierung angelegte Planungssystem, u.a. mit Baurecht und hoheitlicher Administration vor politischem Hintergrund.

Universitäten können aus organisationssoziologischer Sicht als lose gekoppelte Systeme bezeichnet werden, d.h. als „Geflecht relativ autonomer Subeinheiten, die nur okkasionell und nicht in genau spezifizierter Weise untereinander in Verbindung treten“. ${ }^{20}$ Diese polyzentristischen Aktionspotenziale sind Aus-

20 Georg Schreyögg: Organisation. Grundlagen moderner Organisationsgestaltung. 4. vollst. überarb. u. erw. Aufl. Wiesbaden 2003, S. 277. 
druck der Lehr- und Forschungsfreiheit und fördern ein hohes Mass an Selbstbestimmung, beinhalten aber auch die Gefahr der partiellen Entkoppelung und der Überbewertung von Partikularinteressen gegenüber der Gesamtorganisation. „Solche Systeme zeichnen sich durch eine hohe Selbständigkeit ihrer Einzelelemente aus, was zugleich eine geringe Steuerbarkeit und Berechenbarkeit wie auch eine hohe Flexibilität nach sich zieht.“ ${ }^{21}$ Auch Volker Jahr weist darauf hin, dass eine lose Koppelung für Universitäten nicht unbedingt negativ zu werten sei, sondern dass sie „eine ganz eigene Funktionalität [aufweist], auch wenn in einem überzeichneten Bild Hochschulen mitunter gar als Ansammlung von mehreren Dutzend Einzelunternehmen bezeichnet werden, die allein der Umstand verbindet, dass sie sich eine gemeinsame Heizungsanlage teilen“. ${ }^{22}$ Auch wenn internationale Forschungsprogramme, universitäre Forschungsschwerpunkte, fachübergreifende Cluster und hochschulübergreifende Bibliotheksverbünde eine gewisse zentrale Steuerung im fragmentierten Expertensystem erfordern, sind Universitäten als formierte Anarchie ${ }^{23}$ notwendig dezentraler und flexibler angelegt als beispielweise hoheitlich agierende Raumplanungsämter. Wer in internationaler Konkurrenz an den Grenzen des (Nicht-)Wissens arbeitet, muss für die wissenschaftliche Erkenntnisproduktion eigenständig, kurzfristig und flexibel ,bottom up“ agieren können. Dies bedeutet nicht, dass bereitgestellte Infrastrukturen wie Bibliotheken oder Forschungsgeräte verteilt organisiert sein müssen, aber bei historisch gewachsenen Hochschulstrukturen wird die Dezentralität begünstigt. So bilden sich mehrschichtige Bibliothekssysteme heraus und wachsen institutsnah an verschiedenen Standorten in mehr oder weniger geeigneten Immobilien.

Bereits die einfache Unterscheidung zwischen Lehre, Forschung und Verwaltung zeigt auf, dass diese drei Bereiche in ,ihrem Zielsystem, ihrer Struktur, ihrer Ablauforganisation sowie ihren Mitgliedern teilweise inkommensurabel“ ${ }^{24}$ sind und eher als isolierte Subsysteme oder gar Silos betrachtet werden können. Anke Hanft fasst zusammen: „Das Bild der organisierten Anarchie spiegelt die Organisationsdynamik wider, die in akademischen Organisationen immer wieder anzutreffen ist und den Handlungsrahmen für den Einsatz von Managementkon-

21 Klaus Wannemacher, Horst Moog u. a.: ITIL für Hochschulen - Fluch oder Segen? In: Dies. (Hrsg.): ITIL goes University? Serviceorientiertes IT-Management an Hochschulen. Hannover 2008, S. 23-35, hier S. 25.

22 Volker Jahr: Innovation und Macht in der Organisation Hochschule. Kassel 2007, S. 18.

23 Vgl. Michael D. Cohen u. a.: A Garbage Can Model of Organizational Choice. In: Administrative Science Quarterly (Cornell University) 17/1 (1972), S. 1-25, hier S. 1.

24 Wannemacher/Moog u.a., ITIL (wie Anm. 21), S. 25. 
zepten einschränkt. “25 Thomas Hammond beschreibt den Versuch zentrales policy making an einer Forschungsuniversität zu etablieren auch als „Herding Cats“Unterfangen. ${ }^{26}$ So fördert die lose Koppelung dezentral die Forschungs- und Lehrfreiheit und behindert zentral die notwendige Hochschulsteuerung ${ }^{27}$ im Gesamtkontext „multipler Rationalitäten“ ${ }^{28}$ : „What makes the university strong makes it weak.“29

Dem Wissenschaftssystem steht ein Planungssystem gegenüber, das auf Verwaltungsverfahren, Bauvorschriften, Langfristigkeit, legitimierende Partizipation, politische Zentralität und Hierarchie angelegt ist. Auch wenn im Rahmen der Bemühungen des New Public Managements durch wirkungsorientierte Steuerungsmodelle bereits vor geraumer Zeit das von Max Weber beschriebene stahlharte Gehäuse der Hörigkeit bürokratischer Rationalität aufgebrochen wurde, handelt es sich beim Planungssystem im Bereich der Stadt- und Raumentwicklung notwendigerweise um hoheitliche Aufgaben. Während das Agenda-Setting in der Forschung wenige Jahre umfasst, sind planungsrechtliche Grundlagen auf Jahrzehnte ausgelegt. Masterplanungen, Richtpläne und Gestaltungspläne sind weit in die Zukunft reichende Interessensabwägungen und daraus abgeleitet rechtlich bindende Festlegungen. Dies reicht von Verkehrsbaulinien, Höhenkoten, Grünräumen mit Sichtachsen über Vorschriften zur Materialisierung und Denkmalschutz bis hin zu Lärmempfindlichkeitsstufen. ${ }^{30}$ Das bedingt den Einbezug einer Vielzahl von Ämtern und Interessensgruppen, welche zumeist in mehrjährigen Prozessen vom Planungsstart über Test- und Machbarkeitsstudien, interne Vernehmlassungen und öffentliche Auflagen bis hin zu kantonalen Parlamentsbeschlüssen und eidgenössischen Festlegungen koordiniert werden müssen.

25 Anke Hanft: Bildungs- und Wissenschaftsmanagement. München 2008, S. 69.

26 Thomas H. Hammond: Herding Cats in University Hierarchies. Formal Structure and Policy Choice in American Research Universities, In: Roland G. Ehrenberg (Ed.): Governing Academia, who is in charge at the modern university? New York 2004, S. 91-138, hier S. $91 f$.

27 Vgl. Anke Schwank, Marcelo Ruiz: Allokation und Steuerung von Flächenressourcen in Hochschulen. Weimar 2015, S. 49 f.

28 Thomas Schumacher: Strategie und Organisationsdesign in Hochschulen. In: Luzia Truniger (Hrsg.): Führen in Hochschulen. Wiesbaden 2017, S. 69-87, hier S. 72.

29 Susanne Lohmann: Darwinian Medicine for the University. In: Ehrenberg (Ed.), Governing (wie Anm. 26), S. 71-90, hier S. 78.

30 Gino Bätscher: Planungs-, Bau- und Umweltrecht. In: Thomas Wipfler, Adrian Humbel (Hrsg.): Immobilienmanagement. Handbuch für Immobilienentwicklung, Bauherrnberatung, Immobilienbewirtschaftung. 2. Auflage. Zürich 2017, S. 244-283, hier S. $244 f$. 
In diesen starren planungsrechtlichen Rahmen passt sich die Entwicklung von Hochschulimmobilien als eigentliche Bauaufgabe ein. Auch hier muss von der Initiierung von Grossprojekten bis zum Einzug in ein Lernzentrum mit Bibliothek oder ein Laborgebäude ein langer Atem bewiesen werden. Eine Vielzahl von Institutionen aus kantonaler Bildungs- und Baudirektion, Regierung, Parlament, Stadt, Bund, UZH-Gremien, zukünftigen Nutzenden und Planenden stimmen Bauaufgaben über Machbarkeitsstudien, Wettbewerbe und Investitionen untereinander ab. Häufig vergeht eine Dekade bis der umbaute Raum sichtbar wird, wobei die eigentliche Baurealisierung vom Aushub bis zur Inbetriebnahme nur wenige Jahre benötigt. Mit heutigen Nutzenden in einem auf kurzfristiges Agieren angelegten Wissenschaftssystem werden somit Planungsgrundlagen für Gebäude geschaffen, die frühestens in zehn Jahren bezogen werden und Nutzungsdauern für viele Generationen von Forschenden und Studierenden aufweisen.

Wie jedoch ist es um die Zukunft des Buches bestellt? Wie wird in der nächsten Generation unterrichtet? Welche Forschungsmethoden (selbst in den Wirtschaftswissenschaften haben bildgebende Verfahren Einzug gehalten) sind zu erwarten? Verändert die Digitalisierung grundlegend die räumlichen Settings oder geht es vor allem um schnellere Datenverbindungen und höhere Kapazitäten von Rechenzentren? Wurde bereits vor 20 Jahren mit dem Aufkommen von E-Learning das Ende von Hörsälen und physischen Medienbeständen vorausgesagt, werden an vielen Universitätsstandorten auch mit der Intensivierung des digitalen Lernens weiterhin grosse Lernzentren und Bibliotheken gebaut. Fachliche Enkulturation, der persönliche Austausch mit den Peers und die Einbindung in den Wissenschaftsbetrieb werden für Studierende und Forschende auch weiterhin in räumlichen Settings von Hochschulen verortet sein. So verbinden sich digitales Lernen und traditionelle Didaktik mit Hörsälen, Praktikaflächen, Selbststudiumsplätzen und Online-Plattformen zu „Blended Learning“ und „Networked Learning Landscape“. ${ }^{31}$ Die quantitativen und qualitativen Anforderungen an räumliche Ressourcen, wie z.B. Studios für MOOC-Produktionen, Maker Spaces ${ }^{32}$ oder Hörsäle mit Direktübertragung, werden eher zunehmen.

Die Universität Zürich begegnet diesen unterschiedlichen Systemanforderungen aus Akademie und Planung mit einer Reihe von vorausschauenden Massnahmen:

31 Jonas Nordquist: Teaching and Learning Spaces. In: Ian Taylor (Ed.): Future Campus. Design Quality in University Buildings. Newcastle upon Tyne 2016, S. 25-28.

32 Edward M. Corrado: Integrating Technology. In: Marta Mestrovic Deyrub (Ed.): Creating the High-Functioning Library Space. Santa Barbara 2017, S. 114-122. 
- aktive Interessenvertretung im Rahmen der Projektorganisation von Masterplänen und Folgeprozessen (z. B. Hochschulgebiet Zürich Zentrum) ${ }^{33}$

- fachliche Unterstützung von Gebietsplanungen (z. B. Areal Wässerwies)

- Ausarbeiten und Umsetzen von baulich-akademischen Standortstrategien für die UZH (z.B. Bauliche Entwicklungsstrategie Hochschulgebiet Zentrum)

- Bereitstellen von UZH-internen Planungsgrundlagen für Querschnittsthemen (z.B. Projekt UZH Bibliothek der Zukunft, Untersuchung zukünftige Lehrraumbedarfe)

- Gestaltung der baubezogenen Governance (Übernahme der Bauherrenfunktion und teilweise Eigentümervertretung durch UZH)

- Entwickeln einer langfristigen Investitionsplanung für Neubauten und Instandsetzungen

- aktives Portfoliomanagement (Betriebsstrategien, Lebenszyklusbetrachtungen, Rückführung von Wohnraum etc.)

- gezielter und frühzeitiger Einbezug von Nutzenden sowie von externen Experten und Expertinnen bei der Vorbereitung von Bauprojekten (z.B. Bildungs- und Forschungszentrum Wässerwies UZH)

\section{Planungsrechtliche Grundlagen der Bauentwicklung UZH (Zentrum, Irchel)}

Bereits 2009 hat die UZH die Zweistandort-Strategie mit der Fokussierung auf die beiden Standorte Zentrum und Irchel verabschiedet. Aufgrund sehr zurückhaltender kantonaler Investitionen in Grossprojekte für Forschung und Lehre musste mittlerweile neben Zürich Oerlikon als Behelfsstandort für sozialwissenschaftliche Institute mit Büro-, Lehr- und Bibliotheksflächen noch ein weiterer Interimsstandort in Schlieren für biomedizinische Forschungsgruppen mit Laborflächen angemietet werden. Das anhaltende Wachstum der UZH konnte grösstenteils nur durch weitere Belegungsverdichtung in den bestehenden Liegenschaften und Zumietungen gelöst werden. Zusätzlich ist das Immobilienportfolio der UZH durch eine Vielzahl von funktional wenig geeigneten Kleinliegenschaften gekennzeichnet, die noch dazu über das Stadtgebiet verstreut sind. Daraus resultieren betriebliche Ineffizienzen wie weite Wege und aufwendige Bewirtschaftung von vielen

33 Ein Überblick zu den Planungen findet sich auf der Homepage der UZH (http://www.stadt universitaet.uzh.ch/de.html) und des kantonalen Amtes für Raumentwicklung (https://are.zh.ch/ internet/baudirektion/are/de/raumplanung/handlungsfelder/hochschulgebiet_zh.html). 
Einzelliegenschaften. Erschwerend kommt hinzu, dass mittlerweile 40\% der Gebäude in das Inventar des Denkmalschutzes aufgenommen wurden.

Im Rahmen der Zweistandort-Strategie wurde von der Universitätsleitung bereits 2009 u. a. festgelegt: „Ebenfalls sind die Bibliotheken, die derzeit auf über 50 Standorte verstreut sind, soweit sinnvoll und möglich nach Fachgruppen zu arrondieren. Dabei spielt eine Abstimmung mit der Zentralbibliothek sowie die Auslagerung von Beständen in (interkantonale) Speicherbibliotheken eine wichtige Rolle.“34

Mittel- bis langfristig soll die baulich-akademische Hochschulentwicklung an folgenden Zielen ausgerichtet werden:

- Stärkung des Standorts Zentrum durch die Grossprojekte Wässerwies (Lehrund Lernzentrum sowie Forschung mit Büroflächen - FORUM UZH), Gloriarank (biomedizinische Laborflächen) und Schanzenberg (Schwerpunkt Geisteswissenschaften)

- Stärkung des Campus Irchel durch die Initialvorhaben 5. Bauetappe Laborgebäude für Chemie, Instandsetzung der 1. Bauetappe für biologisch-biomedizinische Fächer sowie von Lehrflächen; Arrondierung und Ausbau für die naturwissenschaftlichen und medizinischen Fächer sowie die Veterinärmedizin

- Aufgabe des Ausweichstandorts Oerlikon mit Verlegung der sozialwissenschaftlichen Fächer auf den Campus Irchel (Neubauprojekt)

- Aufgabe des Ausweichstandorts Schlieren mit Verlegung der biomedizinischen Fächer ins Zentrum und auf den Campus Irchel

- Aufgabe von kleinen Liegenschaften in Streulagen und fachbezogene Arrondierung im Zentrum und auf dem Campus Irchel

- Beibehaltung von Standorten aufgrund besonderer Forschungsschwerpunkte (z. B. Agrovet Lindau und Gesundheitscluster Lengg)

34 UZH: Flächenentwicklungsstrategie. Zürich 2009, S. 5. 


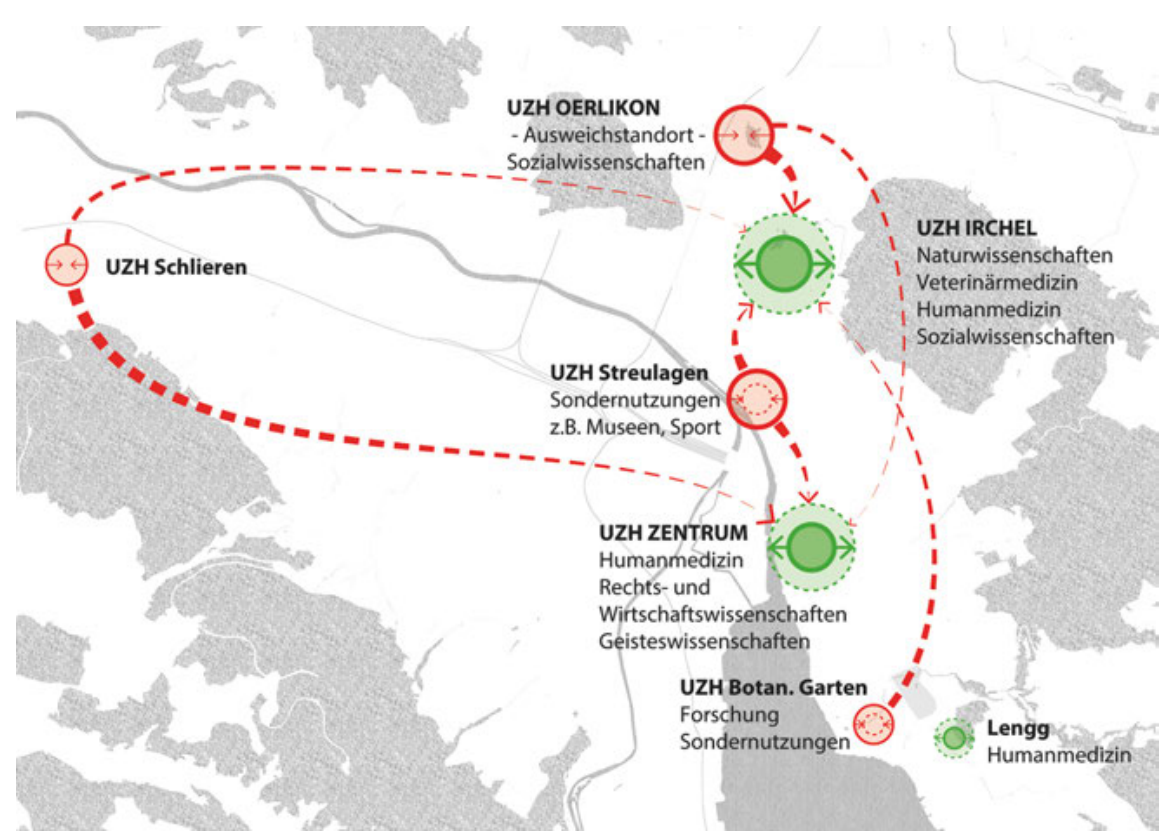

Abb. 3: Zweistandort-Strategie UZH mit Rückführung von Aussenstandorten (eigene Darstellung Immobilienentwicklung UZH, Strategische Planung)

Für das Hochschulgebiet Zentrum wurde eine komplexe Projektorganisation mit Delegierten aus Kanton, Stadt, ETH, USZ und UZH gebildet, um Masterplanung, Vertiefungsstudien, Freiraumplanung und Gestaltungspläne in einem transparenten Rahmen mit den notwendigen Interessensabwägungen vorantreiben $\mathrm{zu}$ können. Die UZH hat für das Zentrum im Jahr 2015 eine eigene bauliche Entwicklungsstrategie verabschiedet. ${ }^{35}$ Hierbei werden u. a. fünf Handlungsfelder (Lehre, Forschung, Dienstleistung, hochschulaffine Nutzungen und Planung) differenziert und mit themenspezifischen Zielsetzungen versehen: „Vorhandene Bibliotheken sollen unter Abgleich des Medienbestands zentralisiert werden. Am Standort Zentrum soll entsprechend eine neue, zentrale Bibliothek geschaffen und primär als Lernzentrum ausgestaltet werden. Parallel dazu soll die Nutzung einer externen Speicherbibliothek weiterentwickelt und ausgebaut werden. “36 Der strategische Anspruch lautet: „Die Bibliotheken der UZH sind zentrale, fach- und fakultätsübergreifende Begegnungsorte, die der Lehre und Forschung dienen.

35 Vgl. UZH: Bauliche Entwicklungsstrategie Universität Zürich, Ausgabe 1: Fokus Standort UZH Zentrum. Zürich 2015.

36 Ebenda, S. II. 
[...] Dezentrale Nebenanlagen werden nur betrieben, wenn aus räumlichen oder fachlichen Gründen eine Einbindung in die zentralen Bibliotheken nicht möglich ist. “37

Für 20\% der Studierenden sollen UZH-weit Selbststudiumsplätze angeboten werden, die jeweils zur Hälfte für leises und lautes Arbeiten vorgesehen sind. Hierbei liegt der Schwerpunkt auf „der Schaffung von modernen Lernumgebungen, anstelle von klassischen Präsenzbibliotheken.“38 Die Zukunft eines Lernzentrums mit Bibliothek wird auf dem Areal Wässerwies mit dem Neubau des Bildungs- und Forschungszentrum FORUM UZH definiert werden. Im Jahr 2017 wurden für das Areal die planungsrechtlichen Grundlagen mit einem Gestaltungsplan festgelegt und für das Bauvorhaben die Durchführung eines Wettbewerbs für das Jahr 2018 vorbereitet. Bei optimalen Planungsbedingungen soll der Neubau FORUM UZH per 2026 realisiert werden und insgesamt rund $35^{\prime} 600 \mathrm{~m}^{2}$ Hauptnutzfläche bereitstellen.

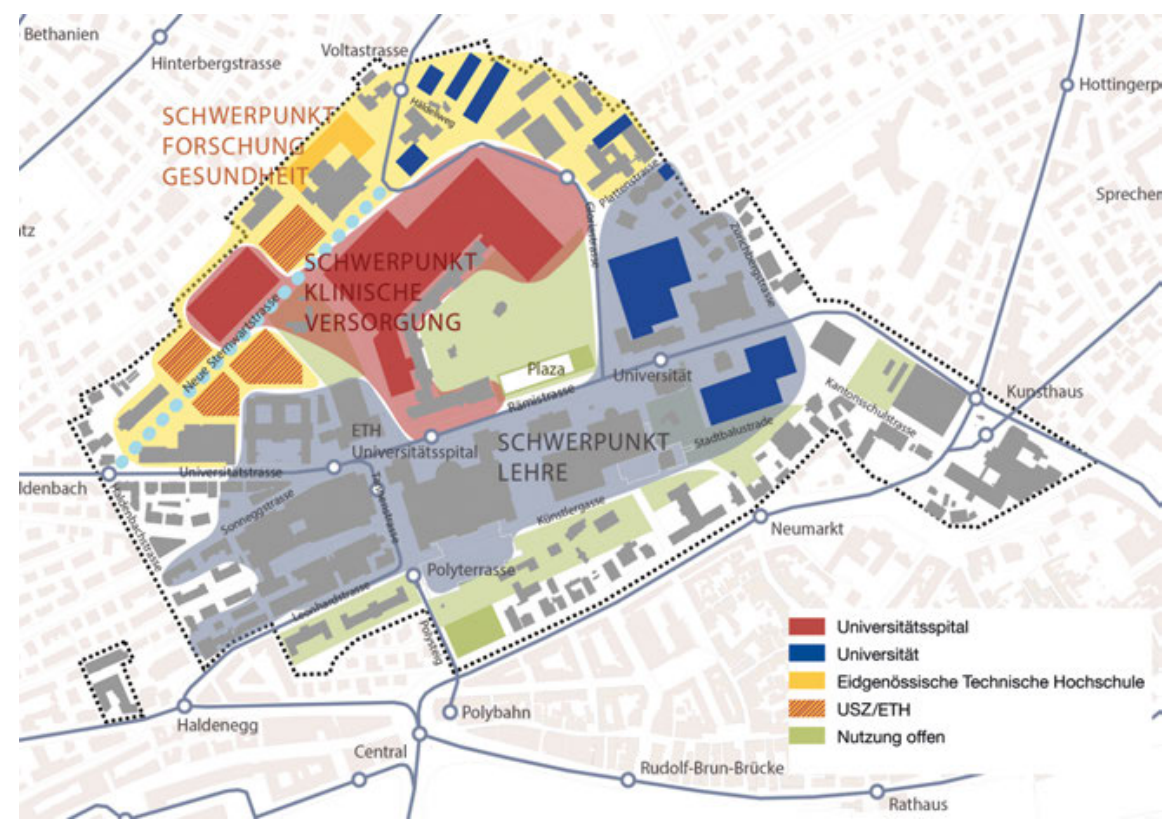

Abb. 4: Planungsperimeter und Schlüsselareale Zentrum (Masterplan Hochschulgebiet Zürich Zentrum) ${ }^{39}$

37 Ebenda, S. 19.

38 Ebenda, S. 12.

39 Kanton Zürich: Masterplan Hochschulgebiet Zürich-Zentrum. Zürich 2014, S. 37. 
Für den Standort Irchel wurden in einem Masterplan ${ }^{40}$ mit Vertiefungsstudie und anschliessendem Richtprojekt ebenfalls die planungsrechtlichen Grundlagen gelegt. Besonders mit der mittel- bis langfristigen Verlegung der sozialwissenschaftlichen Fächer von Oerlikon an den Standort Irchel wird sich auch hier im Rahmen der baulichen Hochschulentwicklung die Frage der Bibliotheksplanung vertieft stellen. Vergleichbar zur baulichen Entwicklungsstrategie mit dem Fokus Zentrum, müssen die Handlungsfelder wie Lehre und Bibliotheken für den Campus Irchel noch weiter definiert werden.

\section{Grossprojekt FORUM UZH als Referenzmodell baulicher Hochschulplanung an der UZH}

Besonders durch die Etablierung eines Lehr- und Lernzentrums mit Medienbereich ist das Bauvorhaben FORUM UZH auf dem Areal Wässerwies für die Zukunft der Bibliotheken ein Ankerpunkt in der Standortentwicklung im Zentrum.

Das Bauvorhaben FORUM UZH muss hierbei auf eine Vielzahl von Anforderungen reagieren:

- Schaffung eines attraktiven Bildungsorts für neue Lernformen durch Etablierung eines Lernzentrums

- Arrondierung von Institutsflächen und Konsolidierung von Forschungsbereichen für Rechts- und Wirtschaftswissenschaften sowie Teilbereiche der Geisteswissenschaften

- Optimierung von Nutzungen durch Konzentration von Gemeinschaftsnutzungen (Bibliotheken und weitere Selbststudiumsplätze)

- Schaffung von Kapazitäten für die hohen Anfängerzahlen in Rechts- und Wirtschaftswissenschaften und die Studierenden der Medizin: intensive Belegung durch fakultätsübergreifende Nutzung von Lehrflächen

- Auflösung von Engpässen bei Selbststudiumsplätzen: Grundlage für zeitgemässes selbstorganisiertes Lernen in Gruppen und als Einzelperson

- Ermöglichung der politisch geforderten Rückgabe von kleineren Immobilien der UZH zur Wohnraumnutzung durch Realisierung von Ersatzflächen: überwiegend Rückgabe von Institutsflächen

- rechtzeitige Bereitstellung von Rochadefläche für die Sanierung des Kollegiengebäudes und für die Baufreiheit auf Entwicklungsarealen: Ersatz für Hörsäle auf den Arealen des Universitätsspitals und Gloriarank

40 Vgl. Ebenda. Ferner: Kanton Zürich: Vertiefungsstudien Campus Irchel. Zürich 2015. 
- Bereicherung des Quartierlebens durch zusätzliche Angebote

- Verbesserung des Sportangebots für Mittelschulen und Akademischen Sportverband

- Gestaltung eines gemeinsamen Hochschulraumes im Verbund mit Kollegiengebäude UZH und Hauptgebäude ETH, der wesentlich zum attraktiven Bildungs- und Forschungsort Zürich mit internationaler Ausstrahlung beiträgt

Als Hauptnutzende stehen Forschende und Studierende im Fokus. Für sie bietet der Neubau FORUM UZH eine optimale Serviceumgebung. Die Forschungs- und Lernumgebung ist ergänzt um unterstützende Nutzungsangebote. Die Vielfalt der miteinander verbundenen Nutzungen macht die Qualität und das Wesen des Projektes aus und soll im Gebäude erlebbar sein.



Abb. 5: Nutzende im Mittelpunkt (Entwurf): Bildungs- und Forschungszentrum FORUM UZH (eigene Darstellung Immobilienentwicklung UZH, Strategische Planung) 


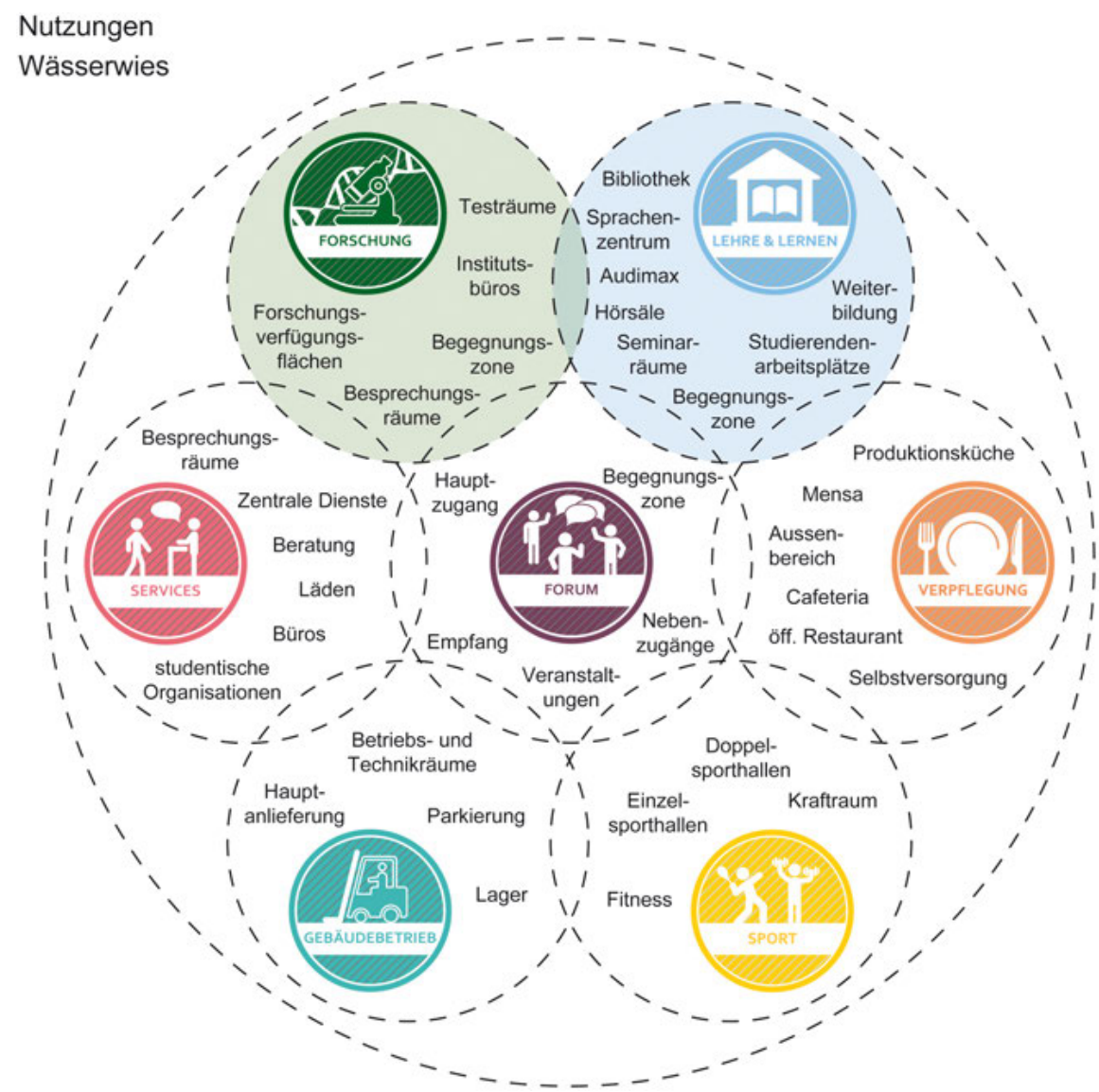

Abb. 6: Funktionsbereiche (Entwurf): Bildungs- und Forschungszentrum FORUM UZH (eigene Darstellung Immobilienentwicklung UZH, Strategische Planung)

Nutzungsbereiche Neubau FORUM UZH auf dem Areal Wässerwies:

- Die zwei treibenden Nutzungsbereiche sind Lehre und Lernen sowie Forschung. Sie werden ergänzt durch eine Serviceumgebung aus den Nutzungsbereichen Forum, Verpflegung, Services, Gebäudebetrieb und Sport.

- Dem zentralen Forum kommt, als Herzstück des Neubaus, inhaltlich wie räumlich eine prominente, vermittelnde Rolle für das Gebäude wie auch für das Umfeld zu. Es repräsentiert die Funktionsbeziehungen und einzelnen Nutzungsbereiche und ist die zentrale Erscheinung der im Gebäude verteilten Kommunikations- und Kollaborationsorte. Als ,akademischer Dorfplatz' fördert das Forum die Verknüpfung der Nutzungsbereiche und ist kommunikativer Dreh- und Angelpunkt für Studierende und Forschende. Da das Forum 
alle Nutzungen verbindet, wurde es auch zum Namensgeber des gesamten Bauvorhabens.

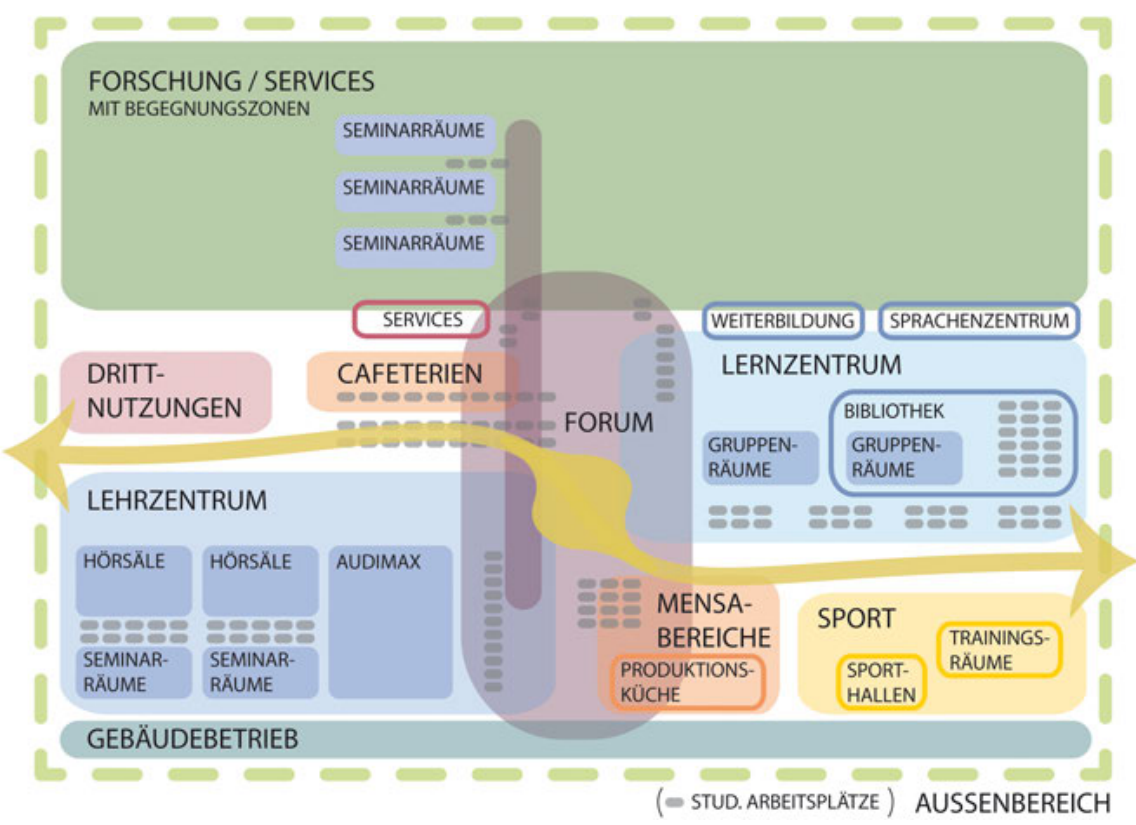

Abb. 7: funktionale Anordnungen (Entwurf): Bildungs- und Forschungszentrum FORUM UZH (eigene Darstellung Immobilienentwicklung UZH, Strategische Planung)

In der funktionalen Verknüpfung der Nutzungsbereiche wird ein Neubau kurzer Wege und vielfältiger Begegnungsorte angestrebt. Forschung und Lehre sind eingebettet in eine Serviceumgebung. Die Bandbreite der Lehr- und Lernorte reicht hierbei von Flächen für das Kontaktstudium (Hörsäle, Seminarräume) bis zum Selbststudium (Gruppen- und Einzelarbeitsplätze). Zur Neugestaltung der Verknüpfung von Kontaktstudium und Selbststudium werden digitale Medien eingesetzt.

Bereich Kontaktstudium:

- Klassische Lehrräume werden weiterhin benötigt, aber um Möglichkeiten für zusätzliche Lehr- und Lernsettings erweitert. So erleichtert die Kombination von Hörsälen und Gruppenarbeitsplätzen den Einsatz spezifischer didaktischer Konzepte.

- Es wird grosser Wert auf interaktive Lehr- und Lernformen gelegt (z.B. Blended Learning, Flipped Classroom, Mixed Reality, Clicker - Classroom Response Systems). Für dynamische Lernprozesse sind unterschiedliche Umgebungen nahe beieinander angeordnet. 
Bereich Selbststudium:

- Die Lehrräume werden durch eine Vielfalt an Selbststudiumsplätzen zu einer komplexen Lernwelt ergänzt.

- Die Lernumgebung im Neubau Wässerwies besteht auch aus einem dezidierten Lernzentrum, das neben zentralen auch dezentrale, institutsnahe studentische Selbststudiumsplätze (für ein individuelles Einzelstudium wie auch selbstorganisierte Gruppenarbeiten) umfasst.

\section{LEHRE \& LERNEN}

LERNZENTRUM MIT BIBLIOTHEK

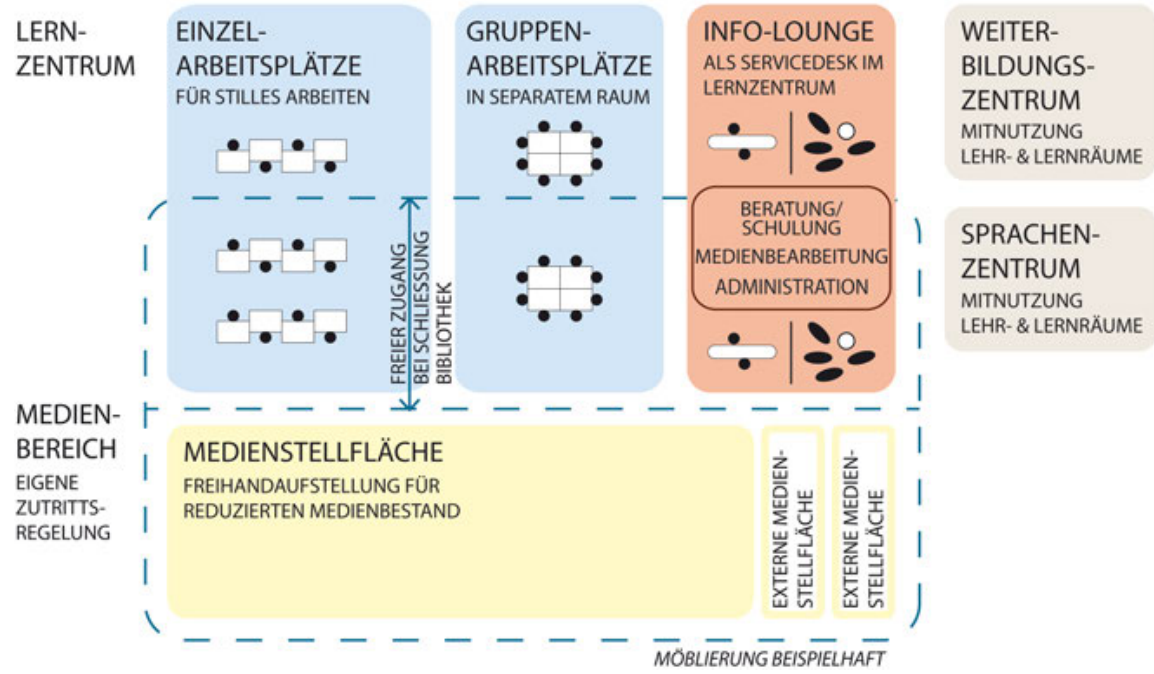

Abb. 8: funktionale Anordnungen (Entwurf): Lernzentrum FORUM UZH (eigene Darstellung Immobilienentwicklung UZH, Strategische Planung)

Das Gebäude FORUM UZH auf dem Areal Wässerwies wird somit den ganzen Zyklus universitären Lehrens und Lernens abbilden: von grossen Vorlesungen für Studienanfänger, über Projektarbeiten und Selbststudium bis hin zu Prüfungen. Es werden Voraussetzungen für die Mitnutzung des Lehr- und Lernzentrums für Weiterbildungsstudierende, öffentliche Vortragsreihen, die Kinder- und Seniorenuniversität oder Volkshochschulkurse geschaffen und so ein umfassender Bildungsort im Zentrum von Zürich etabliert. 


\section{Visionen und Leitideen zur Bibliotheksentwicklung UZH am Beispiel FORUM UZH}

Die mit dem Lernzentrum im FORUM UZH verbundenen Fragen zur Bibliotheksentwicklung stehen in engem Zusammenhang mit den historisch gewachsenen Strukturen der Standorte und der Governance der Bibliotheken an der UZH sowie den organisationssoziologischen Zusammenhängen der Steuerung von Bauprojekten im Kontext von Universitäten.

Bibliotheksentwicklung ist an der Universität Zürich ein zentrales und aktuelles Thema, das auch von der Universitätsleitung vorangetrieben wird. Dies betrifft u.a. die Organisationsentwicklung des bestehenden komplexen dreischichtigen Systems. Hierzu begann im Herbst 2017 ein universitätsweites Projekt UZH Bibliothek der Zukunft, das auf die Schaffung einer neuen, möglichst universitätsintern einheitlichen Organisationsform abzielt. In diesem Rahmen wird als Teilprojekt auch die Standortentwicklung der Bibliothekslandschaft konzeptionell weiterentwickelt. Analysen der Hauptbibliothek zeigen ein erhebliches Optimierungspotenzial auf. ${ }^{41}$ Es werden rund 2,8 Mio. im Bibliotheksverwaltungssystem verzeichnete Bände in den Gebäuden der Universität vorrätig gehalten. Es bestehen erhebliche Redundanzen im Bestand zwischen einzelnen Bibliotheken und auch im Verhältnis zum Angebot der Zentralbibliothek. Die bisherige Bibliotheksentwicklung der UZH verlief insgesamt weitgehend dezentral und am Einzelfall orientiert. Auch wenn bereits erfolgte Arrondierungen wichtige Schritte darstellen, so wurde die 2009 grundsätzlich formulierte Gesamtstrategie im Bereich Bibliotheksentwicklung bisher noch nicht flächendeckend umgesetzt. Die oben aufgezeigten strukturellen organisationssoziologischen Besonderheiten der Universitätssteuerung und -planung sind hier augenfällig und werden durch die besondere Governancesituation eines dreischichtigen Bibliothekssystems verstärkt.

Ein zentraler Anknüpfungspunkt für die Planung der nächsten Jahre ist das Bildungs- und Forschungszentrum FORUM UZH auf dem Areal Wässerwies im Zentrum von Zürich. Auch wenn die genannten planerischen Vorgaben zur Nutzung der Flächen Grenzen setzen, so bietet das Projekt beim derzeitigen Planungsstand mit rund 3'500m² Hauptnutzfläche (HNF) für Bibliotheksbestände (einschliesslich gedruckter Bestände) und weiteren 2'500m² HNF für Plätze zum Selbststudium die Möglichkeit, ein modernes Lernzentrum einschliesslich Stellflächen für Printbestände zu integrieren.

41 Vgl. Wilfried Lochbühler, Simon Allemann: Standortoptimierung Bibliotheken UZH (interner Bericht z.H. Rektorat UZH). Zürich 2016. 
Damit steht der Ansatz im Gesamttrend der Entwicklung der Bibliothek als Ort. Die Dynamisierung hin zu neuen, digitalen Angeboten wird sich in den kommenden Jahren fortsetzen und das bibliothekarische Dienstleistungsangebot weiter verändern. ${ }^{42}$ Diese Entwicklung, die in den Naturwissenschaften bereits weit fortgeschritten ist, wird auch in den Geisteswissenschaften an Fahrt gewinnen. ${ }^{43}$ Bestandesoptimierungen und -reduktionen sind als Pendant dieser Entwicklung inzwischen an zahlreichen Hochschulbibliotheken Usus. ${ }^{44}$ Die Bibliothek als Ort ist grundsätzlich gleichwohl unbestritten und hat in den letzten Jahren an Bedeutung gewonnen. Hierzu haben die Bologna-Reform mit ihren veränderten Anforderungen an das Selbststudium einerseits und die Digitalisierung des Lernens (mit ihren zahlreichen Facetten) andererseits entscheidend beigetragen. Bibliotheken unterstützen durch grosszügige Öffnungszeiten und gehobene technische Infrastruktur für Benutzende verschiedene Settings von Lern- und Arbeitsformen wie Einzel- und Gruppenarbeit, digital unterstütztes Lernen bis hin zu Maker Space-Modellen. Selbstausleihe respektive Selbstverbuchung und professionelle Beratungsangebote runden das Angebot ebenso ab wie Verpflegungsbereiche und Ruhezonen. Weltweit hat im Bibliotheksbau der Gegenwart das ,Learning Center' als Referenzmodell in zahlreichen Differenzierungen und Facetten Einzug gehalten. ${ }^{45}$ Neben häufig zitierten Beispielen wie dem Rolex Learning Center (Lausanne, Schweiz) oder dem Saltire Center (Glasgow, Schottland) haben in jüngster Zeit Bibliotheksbauprojekte in Grossbritannien ${ }^{46}$, Deutschland ${ }^{47}$, in

42 In den Universitätsbibliotheken der Schweiz sind die Ausleihen gedruckter Bestände bisher auf einem konstant hohen Niveau, während die Zugriffe auf elektronische Medien ansteigen. Der Anteil der Erwerbungskosten für elektronische Medien an den Gesamterwerbungskosten stieg von 2011 bis 2016 von 38\% auf 58\% (vgl. Bibliotheksstatistik der Schweiz. Bundesamt für Statistik http://www.bfs.admin.ch - Rubrik: Kultur, Medien, Informationsgesellschaft, Sport).

43 Vgl. z.B. Rudolf Mumenthaler: Herausforderungen für Bibliotheken. In: Ball/Wiederkehr (Hrsg.), Wissen (wie Anm. 10) , S. 85-99.

44 Vgl. den aktuellen Beitrag von Elmar Mittler: Wohin geht die Reise? Bibliothekspolitik am Anfang des 21. Jahrhunderts. In: Bibliothek - Forschung und Praxis 41/2 (2017), S. 213-223, hier S. 220 .

45 Vgl. hierzu die periodisch erscheinenden Berichte New Library Buildings in Europe (2010, 2012, 2014) der LIBER Architecure Group (http://liber-lag.techlib.cz/). Vgl. ferner die einschlägige Fachdatenbank der Hochschule der Medien Stuttgart (https://learning-research.center/data base/).

46 Vgl. Karen Latimer: Architektur für den Zugang: Bibliotheksräume im 21. Jahrhundert neu denken. In: Olaf Eigenbrod, Richard Stang (Hrsg.): Formierungen von Wissensräumen. Optionen des Zugangs zu Information und Bildung. Berlin 2014, S. 37-49.

47 Vgl. Martin Götz: Lernzentren - eine kurze Bestandsaufnahme. In: Bibliothek - Forschung und Praxis 41/1 (2017), S. 100-102. In Deutschland haben zahlreiche Universitätsbibliotheken neue Ansätze realisiert, darunter die UB Mannheim, die UB Bielefeld und die UB Freiburg i.Br. 
den USA $^{48}$, Asien $^{49}$ aber auch in der Schweiz ${ }^{50}$ wesentliche Kernelemente dieses Grundmodells umgesetzt. Es bedeutet aber nicht, dass die papierlose Bibliothek als Leitmodell etabliert ist, vielmehr nehmen Bücher und deren Nutzung (insbesondere in den Geistes- und Sozialwissenschaften) in aktuellen Projekten nach wie vor eine zentrale Rolle ein und werden auf Jahre hinaus zentrale Planungsgrössen bleiben..$^{51}$ Vermehrt digitale Angebote werden die Notwendigkeit, Printmedien vor Ort in Freihandaufstellung anzubieten, künftig jedoch reduzieren. Es ist ein klarer Trend erkennbar, gedruckte Bestände stärker nutzer- und funktionsorientiert zu präsentieren.

Neben den konzeptionellen Aspekten der Planung eines Lernzentrums (s.o.), stellt sich im Fall des Bildungs- und Forschungszentrums FORUM UZH konkret die Frage, welche der bestehenden Bibliotheken bzw. Fachcluster in der Bibliothek auf dem Areal Wässerwies bei begrenztem Raumangebot untergebracht werden können. Konzeptionell zeigen sich innerhalb der UZH durchaus unterschiedliche Einschätzungen, die insbesondere auf den Stellenwert gedruckter Bestände in rund zehn Jahren fokussieren. In der beginnenden Diskussion innerhalb der UZH lassen sich drei grundlegende Modelle unterscheiden:

- Ein konventioneller Ansatz geht von einem weitgehend unveränderten Bedürfnis der Nutzenden, insbesondere der Geisteswissenschaften, an gedruckten Beständen aus und befürwortet die weitgehende Überführung der bestehenden Bestände in Freihandaufstellung. Die Räumlichkeiten der Institutsangehörigen müssen nach dieser Auffassung im Gebäude selbst untergebracht sein, um kurze Wege und optimale Verfügbarkeit jederzeit zu gewährleisten.

- Ein mittlerer Ansatz geht ebenfalls von der Einrichtung einer konventionellen Freihandbibliothek aus, die vor allem häufig genutzte Bestände vor Ort anbietet. Als Planungsgrösse sollen rund ein Drittel der bestehenden Bestände aus Institutsbibliotheken überführt, ein Drittel in die Kooperative Speicherbibliothek ausgelagert und ein Drittel dedoubliert werden. Idealerweise sind die

48 Vgl. Susan McMullen: US Academic Libraries. Today's Learning Commons Models. OECD Publishing, Paris 2008.

49 Ein Beispiel ist die Samsung Library der Sungkyunkwan University in Seol/Südkorea. Vgl. htt p://www.skku.edu.

50 So folgen neben dem Rolex Learning Center der ETH Lausanne auch die neueren Bibliotheken der Zentral- und Hochschulbibliothek Luzern (Uni-PHZ Gebäude), der UB Bern (vonRoll-Areal) oder die Hochschulbibliothek Winterthur der ZHAW (Zürcher Hochschule für angewandte Wissenschaften) diesem Ansatz.

51 Vgl. Bernd Vogel: Flächenplanung für Hochschulbibliotheken. In: Konstanze Söllner, Wilfried Sühl-Strohmenger (Hrsg.): Handbuch Hochschulbibliothekssysteme: Leistungsfähige Informationsinfrastrukturen für Wissenschaft und Studium. Berlin/Boston 2014, S. 471-488, hier S. 477-479. 
Räumlichkeiten der Institutsangehörigen im selben Gebäude, ergänzend wird eine zumutbare Maximaldistanz zwischen Institut und Bibliothek diskutiert. Die Benutzung der Bestände erfolgt entweder in der Bibliothek mit 24 Stunden Zutritt für Dozierende oder durch Ausleihe (Selbstverbuchung). Via Kurierdienst können weniger häufig genutzte Bestände aus der Speicherbibliothek oder aus der Zentralbibliothek angeliefert werden.

- Ein radikalerer Ansatz geht von einem deutlich reduzierten Bedarf an gedruckten Medien bis in zehn Jahren auch in den Geisteswissenschaften aus. Im Rahmen des skizzierten Modells eines Lernzentrums würden nur geringe Printbestände vor Ort präsentiert. Die Nutzung gedruckter Bestände wird überwiegend durch Kurierdienste aus der Zentralbibliothek und der Speicherbibliothek organisiert, wobei das Lernzentrum Wässerwies vor allem eine Rolle als Logistikhub übernimmt. Die Distanz zwischen Institutsräumlichkeiten und Bibliothek spielt in diesem Modell keine entscheidende Rolle mehr.

Im Rahmen des definierten Flächenangebots besteht derzeit noch eine Bandbreite an Vorstellungen für das Lernzentrum mit Bibliothek im Bildungs- und Forschungszentrum FORUM UZH, welche im Laufe der weiteren Projektentwicklung konkretisiert werden müssen. Hier wie in anderen Projekten zur Arrondierung von Bibliotheken bestehen mit der Möglichkeit zur Auslagerung älterer Bestände in die Speicherbibliothek und einer verstärkten Zusammenarbeit mit der Zentralbibliothek als Hub für gedruckte Bestände für die Universität Zürich Chancen, zu einer bedarfsgerechten Anpassung der Bibliotheksflächen zu gelangen.

Das Bauvorhaben FORUM UZH bietet die Chance, die auf dem Campus Irchel bereits fortgeschrittene Arrondierung und Optimierung von Bibliotheksdienstleistungen auch im Zentrum in einem zukunftsgerichteten Modell eines Lehr- und Lernzentrums unter Berücksichtigung der spezifischen Anforderungen geistesund sozialwissenschaftlicher Fachbereiche verstärkt umzusetzen. Es schliesst an die aktuelle Fachdiskussion im Bibliotheksbereich ebenso an wie an aktuelle Entwicklungen im Hochschulbau mit deren spezifischen Rahmenbedingungen. Für eine darüber hinaus gehende Konsolidierung der bestehenden Institute und Bibliotheken im Zentrum bietet zudem das längerfristige Projekt auf dem Schanzenberg Perspektiven. Ebenfalls in längerfristiger Perspektive liegt die Verlegung der sozialwissenschaftlichen Fachbereiche aus Oerlikon auf den Campus Irchel und die damit verbundenen baulichen Massnahmen, für die es erste, zu einem späteren Zeitpunkt noch zu konkretisierende Pläne gibt. Standortoptimierung und Bibliotheksarrondierung wird an der UZH noch für Jahrzehnte ein Schlüsselthema bleiben. 